\title{
Efficient Multiuser Receivers for CDMA Systems
}

\author{
Chaitali Sengupta ${ }^{\dagger} \quad$ Suman Das Joseph R. Cavallaro Behnaam Aazhang \\ \{chaitali,suman,cavallar,aaz\}@rice.edu \\ Department of Electrical and Computer Engineering, Rice University. \\ 6100 South Main Street, MS 366 \\ Houston, TX 77005, USA.
}

\begin{abstract}
We focus on the design of multiuser receiver structures for Code Division Multiple Access (CDMA) communication systems, in the presence of multipath effects and multiple sensors at the base station receiver. We present a flexible and extensible framework that allows the use of an estimated effective spreading code from the channel estimation phase, in the multiuser detection process. The effective spreading code captures all the channel parameters such as path delays, attenuation factors, and directions of arrival. Hence estimation of this one composite vector removes the necessity of estimating each individual parameter, thus reducing computational complexity. Our results also show that this approach leads to better performance for multiuser detection, especially when the channel consists of a number of low energy paths in addition to a few discrete strong paths.
\end{abstract}

\section{Introduction}

In CDMA communication systems the different mobile users are distinguished at the base station receiver by the unique spreading codes assigned to the users. The binary antipodal $( \pm 1)$ information bits are modulated by the code waveforms before transmission. The received signal is a linear superposition of the multiple copies of the signals transmitted by all the users due to multipath effects, each path attenuated by arbitrary factors and delayed by an arbitrary amount.

The performance of the conventional single user receiver is limited by the near-far effect and more generally by the multiple access interference. Centralized power control can be used to somewhat reduce the near-far effect but the error performance is still far from optimum as was shown by Verdú in [11]. Verdú's work was followed by many suboptimal near-far resistant detectors of lower computational complexity (for overview see [5], [12]). However, these methods deal only with detection and require accurate estimates of the various channel parameters, such as multipath delays and attenuation. Several multiuser channel estimation techniques have been proposed in the literature [1], [10], [8], [4], [15], [7] which are suitable for channel estimation in a wide range of scenarios.

Traditionally, channel estimation and detection have been considered as two distinct and separate modules in the receiver. It has been assumed that the channel estimator provides estimates of the channel delays and amplitudes and the detector uses these estimates to process the received signal to detect the transmitted symbols. However, there are certain advantages that can be gained from jointly designing the channel estimator and detector. Recently, several researchers [14], [13], [6] have approached

$\dagger$ Currently at Texas Instruments, Inc. Dallas, TX

This work was supported in part by Nokia Corporation, by the Texas Advanced Technology Program under grant 1997-003604-044, and by NSF under grant NCR-9506681 and CDA-9617383. this problem by using a combination of various multiuser techniques, such as the subspace-based approach for both channel estimation and detection. The advantages of such joint approaches include gains in performance as well as reduction in computation costs.

The contributions of this paper are twofold. First, we present a system framework that facilitates joint channel estimation and detection in a scenario involving multiple users, multiple paths, and multiple sensors at the receiver. It defines an effective spreading code [13] vector for each user which captures all channel parameters, such as path delays, attenuation factors, and directions of arrival. This framework allows us to estimate the effective spreading code and use it directly in the detection process without estimating each individual channel parameter. In addition, it allows usage of the same chip matched filter output for channel estimation as well as detection. The chip matched filter output does not need to be recomputed for the detection step after the path delays have been estimated in the channel estimation stage. The framework is flexible enough to allow application of any channel estimation scheme (e.g. subspace-based or maximum likelihood) to estimate the effective spreading code as well as any multiuser detector.

The second contribution of this paper is a particular joint channel estimation and detection scheme based on the above framework. The scheme uses a maximum likelihood approach [7], [9] to estimate the effective spreading code which is then fed into a multiuser detector based on the decorrelating detector. This technique is less computationally complex than the subspace-based joint approaches [13], [14], which require an expensive subspace decomposition step. Our simulation results show the performance gains from such a system, especially when the channel consists of a number of low energy paths in addition to a few discrete strong paths. Our complexity estimates illustrate the savings in computation that can be expected from our maximum likelihood based channel estimation scheme, when the effective spreading code is being estimated instead of each individual channel parameter.

\section{CDMA System Model : Multipath And Multiple Sensors}

We assume a $K$-user direct sequence CDMA system with BPSK (Binary Phase Shift Keying) modulation with each transmitted signal selected from a binary alphabet and limited to $[0, T]$, where $T$ is the symbol period. The complex baseband representation of the $k^{\text {th }}$ user's transmitted sig- 
nal is given by

$$
s_{k}(t)=\sqrt{P_{k}} \sum_{i=1}^{N} b_{k, i} c_{k}(t-i T),
$$

where $P_{k}$ is the transmitted power, $b_{k, i} \in\{+1,-1\}$ is the $i^{\text {th }}$ transmitted bit and $c_{k}(t)$ is the spreading waveform. The parameter $N$ denotes the number of bits being considered. The spreading or code waveform is composed of $N_{c}$ chips and if we assume BPSK for the spreading modulation we have $c_{k}(t)=\sum_{n=0}^{N_{c}-1} c_{k, n} \Pi\left(t-n T_{c}\right)$, where $c_{k, n} \in\{+1,-1\}$ and the chip pulse waveform $\Pi(t)$ is a rectangular pulse of duration $T_{c}$. We will assume that the extent of the spreading code is one bit period and hence we have $T=N_{c} T_{c}$.

It has been shown [4] that using an antenna array at the receiver leads to considerable performance gains due to spatial diversity. However, this requires channel estimation at each of the sensors and application of this extra channel information to the detection process. Hence, we have incorporated this feature in our system framework for joint channel estimation and detection. The front end of the receiver consists of an antenna array of $M$ sensors arranged in a specific geometry. The corresponding array response vector, which is the response to a propagating plane wave impinging on the array at an angle $\theta$ is given by $\left[\rho^{(1)}(\theta), \cdots, \rho^{(m)}(\theta), \cdots, \rho^{(M)}(\theta)\right]$, and is determined by the geometry of the array. In this paper, no a priori assumption is made on the geometry of the array. However it is assumed that the time taken by the signal to traverse the physical array is much smaller than the inverse of the message bandwidth and hence, the envelope characteristics of the signal do not vary across the array.

Let us assume, here, that the channel for each user consists of $P$ distinct and resolvable propagation paths [1]. The impulse response of the channel seen by user $k$ is given by $h_{k}(t)=\sum_{p=1}^{P} w_{k, p} \delta\left(t-\tau_{k, p}\right)$ where, $w_{k, p}$ is the complex amplitude with which the $p^{\text {th }}$ path of the $k^{\text {th }}$ user is received and includes contributions from the channel attenuation and the phase offset and $\tau_{k, p}$ is the relative delay with respect to a reference at the receiver. The channel parameters are assumed to be unknown but constant during the estimation process. It may be noted that the assumption of $P$ distinct paths is not essential for the development of our algorithm. It is introduced here only to illustrate the nature of the channel. In fact, we will show later that the number of paths do not affect the size of the problem or the structure of the algorithm.

Accordingly the received signal at the base station is a superposition of multiple copies of attenuated and delayed signals transmitted by all the $K$ users. Therefore, the signal at the $m^{t h}$ sensor is given by:

$$
r^{(m)}(t)=\sum_{k=1}^{K} \sum_{p=1}^{P} w_{k, p} s_{k}\left(t-\tau_{k, p}\right) \rho^{(m)}\left(\theta_{k, p}\right)+\nu^{(m)}(t) .
$$

The additive noise is assumed to have some correlation across the array and is not necessarily white.
The continuous time signal at each sensor is then discretized [1] by sampling the output of a chip-matched filter, at the chip rate. Next, observation vectors are formed by collecting together $N_{c}$ successive outputs of the chip matched filter $r^{(m)}[n]$. Each observation vector corresponds to a time interval equal to one bit period.

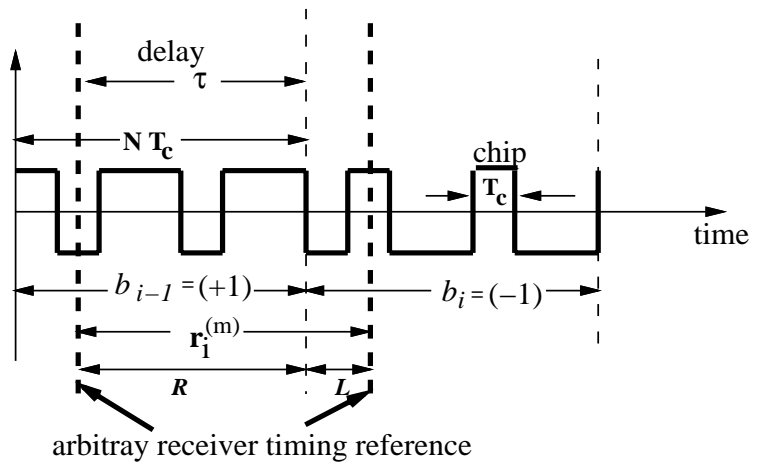

Fig. 1. System model - received signal.

The system is asynchronous and the receiver has an arbitrary timing reference which will not be aligned to actual transmitted bit boundaries. Hence, each observation vector can be viewed as a linear combination of $2 K$ signal vectors - two components from each user due to the past and current bits as shown in Figure 1. In Figure 1, the received vector $\mathbf{r}_{i}^{(m)}$ overlaps the two adjacent transmitted bits $b_{i-1}$ and $b_{i}$. So the contribution of the spreading code of the user to vector $\mathbf{r}_{i}$ appears in two parts - the right part (shown as $R$ ) of bit $b_{i-1}$ and the left part (shown as $L$ ) of bit $b_{i}$. Expressing $\mathbf{r}_{i}^{(m)}$ in terms of its various components:

$$
\mathbf{r}_{i}^{(m)}=\mathbf{A}^{(m)} \mathbf{b}_{i}+\nu_{i}^{(m)}, \quad \nu_{i}^{(m)} \sim \mathcal{N}\left(\mathbf{0}, \mathcal{K}^{(m)}\right)
$$

We denote the $i^{\text {th }}$ bit of the $k^{\text {th }}$ user by $b_{k, i}$ and the vector $\mathbf{b}_{i}=\left[b_{1, i-1}, b_{1, i}, \ldots, b_{k, i-1}, b_{k, i}, \ldots, b_{K, i-1}, b_{K, i}\right]^{\top}$. Let us consider the columns of the matrix $\mathbf{A}^{(m)}$. As explained in Figure 1, the contribution of the spreading code of the user to vector $\mathbf{r}_{i}$ appears in two parts - $R$ and $L$. Hence, matrix $\mathbf{A}^{(m)}$ has columns corresponding to these two parts: denoted by the superscripts $R$ and $L$ and is given by $\mathbf{A}^{(m)}=\left[\begin{array}{lllll}\mathbf{a}_{1}^{R} & \mathbf{a}_{1}^{L} & \cdots & \mathbf{a}_{K}^{R} & \mathbf{a}_{K}^{L}\end{array}\right]$.

Each of the columns $\mathbf{a}_{k}^{R}$ and $\mathbf{a}_{k}^{L}$ for each user $k$, are functions of the corresponding delays $\left(\tau_{k, 1}\right.$ to $\left.\tau_{k, P}\right)$, attenuation factors $\left(w_{k, 1}\right.$ to $\left.w_{k, P}\right)$, and array responses $\left(\rho_{k, 1}^{(m)}\right.$ to $\left.\rho_{k, P}^{(m)}\right)$. Here, $\rho_{k, p}^{(m)}=\rho^{(m)}\left(\theta_{k, p}\right)$.

If $T_{c}$ is the chip period, let $\tau_{k, p} / T_{c}=q+\gamma, q \in$ $\left\{0,1, \cdots, N_{c}-1\right\}, \gamma \in[0,1)$, we have [1]:

$$
\begin{aligned}
\mathbf{a}_{k}^{R} & =\sum_{p=1}^{P}\left[w_{k, p}\left\{(1-\gamma) \mathbf{c}_{k}^{R}[q]+\gamma \mathbf{c}_{k}^{R}[q+1]\right\} \rho_{k, p}^{(m)}\right] \\
\mathbf{a}_{k}^{L} & =\sum_{p=1}^{P}\left[w_{k, p}\left\{(1-\gamma) \mathbf{c}_{k}^{L}[q]+\gamma \mathbf{c}_{k}^{L}[q+1]\right\} \rho_{k, p}^{(m)}\right]
\end{aligned}
$$

where $\mathbf{c}_{K}^{R}[q]$ and $\mathbf{c}_{K}^{L}[q]$ are the spreading codes shifted by 
integer (multiples of chips) delays.

$$
\begin{aligned}
\mathbf{c}_{k}^{R}[q] & =\left[c_{k, N_{c}-q} \cdots c_{k, N_{c}-1} 0 \cdots 0\right]^{\top} \\
\mathbf{c}_{k}^{L}[q] & =\left[0 \cdots 0 c_{k, 0} \cdots c_{k, N_{c}-q-1}\right]^{\top}
\end{aligned}
$$

We now have a model describing the discretized received signal at each sensor. It may be noted that the size of $\mathbf{a}_{k}^{R}$ or $\mathbf{a}_{k}^{L}$ (and hence $\mathbf{A}^{(m)}$ ) does not depend on the number of paths. As the number of paths increase, these vectors (and matrix) becomes more dense but does not increase in size.

We then combine the observations at all the sensors so that we can later exploit temporal as well as spatial diversity. Hence, the observation vector $\mathbf{r}_{i}$, of length $M N_{c}$, across the array, $\mathbf{r}_{i}=\left[\mathbf{r}_{i}^{(1)^{\top}}, \cdots \mathbf{r}_{i}^{(k)^{\top}}, \cdots \mathbf{r}_{i}^{(K)^{\top}}\right]^{\top}$ is

$$
\mathbf{r}_{i}=\mathbf{A} \mathbf{b}_{i}+\nu_{i}
$$

where the noise vector $\nu_{i}$ is formed from the components $\nu_{i}^{(m)}$ and is assumed to have an unknown covariance of $\mathcal{K}$. The matrix $\mathbf{A}$ is composed of the matrices $\mathbf{A}^{(m)}, m=$ $1, \cdots, M$ and is a function of the spreading codes of all the users as well as all the channel parameters corresponding to all the sensors.

In [7], this model is developed further. The structure of matrix $\mathbf{A}$ is exploited in order to separate it into two matrices. One matrix, $\mathcal{U}$, is a function of the spreading codes and the other $\mathbf{Z}$ consists of all the unknown channel parameters of all the paths of all the users at all the sensors. So, the received signal across all the sensors is:

$$
\mathbf{r}_{i}=\mathcal{U} \mathbf{Z} \mathbf{b}_{i}+\nu_{i}
$$

The structure $(\mathcal{U} \mathbf{Z})$ shows the reason why the matrix $\mathbf{A}$ is referred to as the effective spreading code matrix - it is the output of the channel when the input is the spreading codes of all the users. In other words, it encapsulates the effect of the channel on the spreading codes. The matrix $(\mathcal{U Z})$ is constructed from 2 components $(R$ and $L)$ for each user:

$$
\mathbf{r}_{i}=\left[\begin{array}{lll}
\mathcal{U}_{1}^{R} \mathbf{z}_{1} & \mathcal{U}_{1}^{L} \mathbf{z}_{1} \cdots \mathcal{U}_{K}^{R} \mathbf{z}_{K} & \mathcal{U}_{K}^{L} \mathbf{z}_{K}
\end{array}\right] \mathbf{b}_{i}+\nu_{i}
$$

where $\mathcal{U}_{k}^{R}$ and $\mathcal{U}_{k}^{L}$ are matrices of the spreading codes of the $k^{t h}$ user and the vector $\mathbf{z}_{k}$ is composed of all the channel parameters of the same user.

\section{Algorithm DESCRIPTION}

In the rest of the paper, we will develop the joint channel estimation and detection scheme, which will first estimate the matrix $\mathbf{A}$ using the maximum likelihood approach and then directly use this estimate in the detection step, without first extracting the individual channel delays and attenuation factors from $\mathbf{A}$.

Using the maximum likelihood estimate of the effective spreading code, $(\mathcal{U} \mathbf{Z})$, in detection as opposed to feeding back the maximum likelihood estimate of the individual parameters into the detection step has two advantages. First, we can save any error arising from the process of fitting the estimated $(\mathcal{U} \mathbf{Z})$ to the model for $(\mathcal{U} \mathbf{Z})$ as a function of the individual parameters. This process would be necessary for extracting the parameters from the estimated $(\mathcal{U} \mathbf{Z})$. This reduction in channel estimate error will lead to improvement in bit-error rate performance of the detectors. We would also save the computation cost that would be required to perform the extraction of the channel parameters.

\section{A. The Channel Estimation Algorithm}

Given $N$ observations $\mathbf{r}_{1}, \mathbf{r}_{2}, \ldots, \mathbf{r}_{N}$ the log-likelihood function $(\Lambda)$ of these observations can be expressed as

$$
-\ln |\mathcal{K}|-\operatorname{tr}\left\{\frac{1}{\mathrm{~N}} \sum_{\mathrm{i}=1}^{\mathrm{N}}\left(\mathbf{r}_{\mathrm{i}}-\mathbf{A} \mathbf{b}_{\mathrm{i}}\right)^{\mathrm{H}} \mathcal{K}^{-1}\left(\mathbf{r}_{\mathrm{i}}-\mathbf{A} \mathbf{b}_{\mathrm{i}}\right)\right\}
$$

where $|\cdot|$ represents the determinant operator and $\operatorname{tr}(\cdot)$, the trace operator. Maximization of the log-likelihood function is carried out with respect to $\mathbf{A}$, following the steps outlined in [7]. The optimum effective spreading code should satisfy $E(\mathbf{A b})=E(\mathbf{r})$. The known preamble $\mathbf{b}$ is transmitted to estimate the optimal effective spreading code $\mathbf{A}=E\left(\mathbf{r} \mathbf{b}^{\top}\right)\left(E\left(\mathbf{b b}^{\top}\right)\right)^{-1}$.

\section{B. Multishot Multiuser Linear Detection}

Linear multiuser detection requires the output of codematched filters for each user. All earlier efforts in this area assume that the code-matched filters require alignment of the processing window of the code-matched filter to bit boundaries of each user. Hence the chip matched filters had to be essentially recomputed for each user after the delays were estimated and fed back, in order to align the processing window of the code-matched filters to bit boundaries of each user. This meant that the chip matched filters used for channel estimation that were not aligned to bit boundaries could not be used to generate the code-matched filter outputs.

The use of the framework described in the previous section does not require such re-alignment and recomputation of the chip matched filter. This is because the delay information is embedded within the effective spreading code and the detection process described later in this section does not require that the processing window of the codematched filter be aligned to bit boundaries.

Moreover, previous efforts that have used a similar approach [2], [9] are essentially single shot detectors. Since the contribution of bit $\mathbf{b}_{i}$ extends over two received signal periods, $\mathbf{r}_{i}$ and $\mathbf{r}_{i+1}$, two estimates of $\mathbf{b}_{i}$ are obtained from them. Finally a maximal gain ratio combining technique is used to arrive at the final measure. Single-shot detection implies that the detector deals with one symbol period at a time and does not account for the fact that two consecutive symbols of each interferer overlap with each symbol of the desired user. Performance gains may be expected if this asynchronous nature [5] of the system can be accounted for by detecting a block of bits for all the users simultaneously. In addition, the multishot detector is near-far resistant as it accounts for the interference from both overlapping symbols of the interfering users.

In this section we will present a scheme for such multishot detection. The model for the received signal at an 
antenna array is given by $\mathbf{r}_{i}=\mathcal{U} \mathbf{Z} \mathbf{b}_{i}+n u_{i}$, where $\mathbf{b}_{i}=$ $\left[b_{1, i-1}, b_{1, i}, \ldots, b_{K, i-1}, b_{K, i}\right]^{\top}$. Rearranging the columns and the vector we can write

$$
\begin{gathered}
\mathbf{r}_{i}=[\underbrace{\left(\mathcal{U}_{1}^{R} \mathbf{z}_{1}\right) \cdots\left(\mathcal{U}_{K}^{R} \mathbf{z}_{K}\right)}_{\mathbb{U}^{R}} \underbrace{\left(\mathcal{U}_{1}^{L} \mathbf{z}_{1}\right) \cdots\left(\mathcal{U}_{K}^{L} \mathbf{z}_{K}\right)}_{\mathbb{U}^{L}}]\left[\begin{array}{c}
\vdots \\
b_{K, i-1} \\
b_{1, i} \\
\vdots \\
b_{K, i}
\end{array}\right] \\
+\nu_{i} .
\end{gathered}
$$

In order to do multishot detection, the above model should be extended to include multiple bits. Let us assume that we will consider $D$ bits at a time $(i=1,2, \cdots, D)$. So, we form the multishot received vector $\mathbf{r}$ of length $M N_{c} D$ by concatenating $D \mathbf{r}_{i}$-s $(i=1,2, \cdots, D)$. The vector $\mathbf{r}$ can be written as:

$$
\mathbf{r}=\left[\begin{array}{ccccc}
\mathbb{U}^{R} & \mathbb{U}^{L} & 0 & 0 & 0 \\
0 & \mathbb{U}^{R} & \mathbb{U}^{L} & 0 & 0 \\
\vdots & \ddots & \cdots & \ddots & \vdots \\
0 & 0 & 0 & \mathbb{U}^{R} & \mathbb{U}^{L}
\end{array}\right]\left[\begin{array}{c}
b_{1,1} \\
\vdots \\
b_{K, 1} \\
\vdots \\
b_{1, D} \\
\vdots \\
b_{K, D}
\end{array}\right]+\nu
$$

The model can be written in more compact matrix form as $\mathbf{r}=\mathcal{A} \mathbf{b}+\nu$. Using this model for the received signal, equations (4) for the matched filter and (5) for linear detectors may be applied to $\mathbf{r}$ to achieve multishot detection,

$$
\mathbf{y}=\mathcal{A}^{H} \mathbf{r},
$$

where $\mathbf{y} \in \mathbb{C}^{2 K D}$. Since all the delay and angle of arrival informations are captured in $\mathcal{A},(4)$ can be interpreted as a code-matched filter operation, followed by an 'anglematched' filter or conventional beam-former. The linear detector $G \in \mathbb{C}^{2 K \times 2 K}$ will produce the final decision statistics for all the users as

$$
\mathbf{x}_{i}=G \mathbf{y}_{i}=G \mathcal{A}^{H} \mathbf{r}_{i} .
$$

The required bit estimates are the hard decision: $\hat{\mathbf{b}}_{i}=$ $\operatorname{sign}\left(\mathbf{x}_{i}\right)$. The computational complexity to solve the linear system given by (5) can be reduced by using techniques presented in [3].

The detection scheme presented above incorporates the benefits of multiple paths, as well as multishot detection. Since the effective spreading code $\mathcal{A}$ is directly used in the detection process instead of first extracting the individual parameters, we avoid any error that would arise from the parameter extraction process.

\section{Results}

We conducted several experiments to study the performance of our algorithm. The base asynchronous system consists of 15 users using Gold code of length 31 as spreading waveform sequences. The weakest user is $0-10 \mathrm{~dB}$ weaker than the other users and the results are presented for the weakest user.

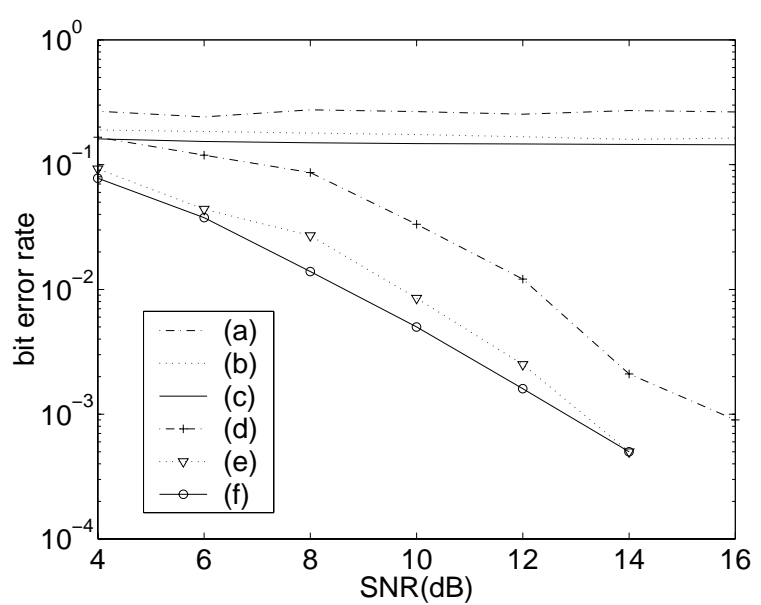

Fig. 2. Single path, single sensor case: BER from (I) matched filter using (a) estimated individual parameters, (b) estimated effective spreading code and (c) true parameters or perfect channel knowledge; and (II) decorrelator using (d) estimated parameters, (e) estimated effective spreading code and (f) true parameters

Figure 2 shows that the performance improves by $2-4 \mathrm{~dB}$ as we go from extracting individual parameters to estimating the effective spreading code only in the case of a multishot multiuser detector. There is slight improvement even in the performance of a matched filter which signifies the improvement in the channel estimation process. We have also presented the result of the detectors when the channel is known perfectly and it shows how accurate the channel estimation technique is.

Our framework is flexible enough to support multiple paths and multiple sensors and in fact there is considerable gain that can be expected by using multiple sensors as shown in [4]. We used three sensors and three paths with the secondary paths having energy $1 / 2$ and $1 / 3$ of the principal path. It should be noted that the previous studies did not consider multiple paths and multiple sensors along with a multishot detector. Figure 3 shows the gain in performance that we can expect by employing multiple sensors along with the effective spreading code scheme in a multipath scenario.

However the most significant performance gain is seen in a more realistic scenario. Researchers have so far tried to model a physical channel by a finite number of multipaths. In fact studies indicate that there are only a finite number of paths with significant strength. However there will be many other paths with relatively insignificant strength. The loss in performance by ignoring these minor paths in a conventional receiver is insignificant. However, in the case of a multiuser receiver, significant performance gains can be achieved by using the effective spreading code scheme when compared with a channel model with finite number of paths and estimating and using the channel parameters for these paths. Figure 4 shows the improvement in performance for such a system. The system has 3 major paths as before but with two other minor paths each of strength $1 / 50^{t h}$ of the direct path. The estimation algorithm that extracts the individual parameters ignores the two small paths. The gain in performance is more dramatic in this 


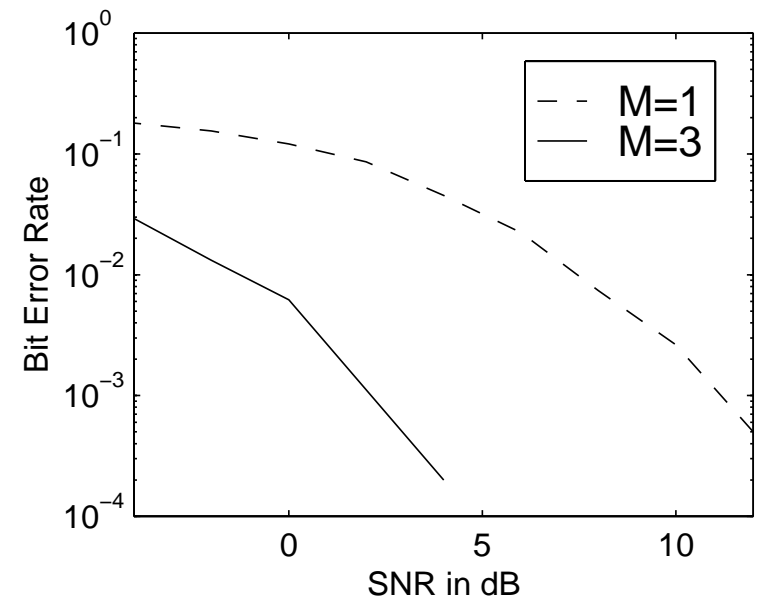

Fig. 3. Multipath, multiple sensors case: BER from multishot decorrelator with estimated effective spreading code and 1 and 3 sensors.

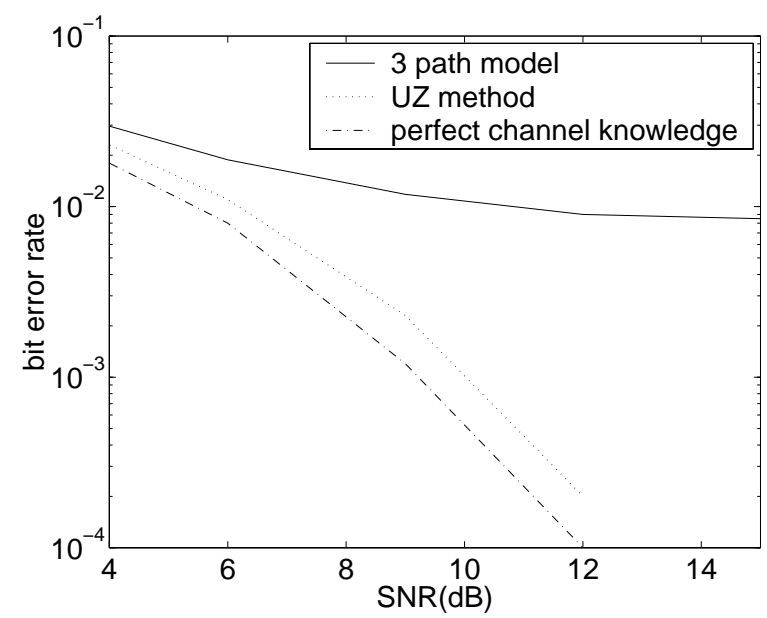

Fig. 4. Performance of the system when the number of multipath components is not known exactly

scenario.

Our receiver not only provides gains in performance but also a reduction in the amount of computation required. Using the joint channel estimation and detection scheme it is possible to avoid the computation to estimate $\mathbf{z}_{k}$ and to extract the parameters from $\mathbf{z}_{k}$. The computational complexity required to calculate the effective spreading code and the extra computation required to extract the parameters are detailed in Table I. The Table shows the workload in the acquisition phase only, when the preamble is being transmitted. The second and third row details those computations that can be avoided by using the effective spreading code for the detectors.

\section{Conclusion}

We have proposed a new joint channel estimation and detection scheme for CDMA systems. We claim that it is more effective to estimate the effective spreading code rather than modeling and estimating the channel as discrete multipath components. We have also shown that it is possible and indeed beneficial to use the estimated received effective spreading code directly in detection, both in terms
TABLE I

Complexity Reduction: The second and third rows show the computation that can be avoided by using the new algorithm

\begin{tabular}{||c|c|}
\hline \hline Processing & Complexity \\
\hline \hline Estimate $\mathcal{U} \mathbf{Z}$ & $\begin{array}{c}O\left(M N_{c} K N\right), O\left(K^{2} N\right) \\
O\left(M N_{c} K^{2}\right)\end{array}$ \\
\hline \hline Estimate $\mathbf{z}_{k}$ & $O\left(M^{2} N_{c}^{2} K\right)$ \\
\hline $\begin{array}{c}\text { Extract } \\
\text { parameters }\end{array}$ & $O\left(M N_{c} K\right)$ \\
\hline \hline
\end{tabular}

of performance as well as reduced computation load.

\section{REFERENCES}

[1] S. E. Bensley and B. Aazhang. Subspace-based channel estimation for code division multiple access communication systems. IEEE Trans. Communications, 44(8):1009-1020, August 1996.

[2] S. E. Bensley and B. Aazhang. Maximum likelihood synchronization of a single user for CDMA communication systems. IEEE Trans. Communications, 46(3):392-399, March 1998.

[3] S. Das, J. R. Cavallaro, and B. Aazhang. Computationally Efficient Multiuser Detectors. In 8th IEEE International Symposium on Personal,Indoor, and Mobile Radio Communications (PIMRC), pages 62-67, 1997. Helsinki, Finland.

[4] R. Madyastha and B. Aazhang. Antenna arrays for joint maximum likelihood parameter estimation in CDMA systems. Conference on Information Sciences and Systems, II:984-988, March 1997. Baltimore, MD.

[5] S. Moshavi. Multi-user detection for DS-CDMA communications. IEEE Communications Magazine, pages 124-136, October 1996.

[6] S. Parkvall, E. G. Strom, L. B. Milstein, and B. E. Ottersten. Asynchronous near-far resistant DS-CDMA receivers without a priori synchronization. IEEE Trans. Communications - To appear, 1998.

[7] C. Sengupta, J. R. Cavallaro, and B. Aazhang. Maximum likelihood multipath channel parameter estimation in CDMA systems using antenna arrays. IEEE International Symposium on Personal, Indoor, and Mobile Radio Communications (PIMRC), pages 1406-1410, September 1998. Boston, MA.

[8] C. Sengupta, J. R. Cavallaro, and B. Aazhang. Subspace-based tracking of multipath channel parameters for CDMA systems. European Trans. Telecommunications, Special issue on CDMA techniques for wireless communication systems, 9(5):439-447, Sep.-Oct. 1998.

[9] C. Sengupta, A. Hottinen, J. R. Cavallaro, and B. Aazhang. Maximum likelihood multipath channel parameter estimation in CDMA systems. Conference on Information Sciences and Systems, March 1998. Princeton, NJ.

[10] E. G. Strom, S. Parkvall, S. L. Miller, and B. E. Ottersten. DSCDMA synchronization in time-varying fading channels. IEEE Journal on Selected Areas in Communication, pages 1636-1642, Oct. 1996.

[11] S. Verdú. Minimum probability of error for asynchronous Gaussian multiple-access channels. IEEE Trans. Information Theory, IT-32:85-96, Jan. 1986.

[12] S. Verdu. Multiuser detection. Cambridge University Press, 1st edition, 1998. New York, NY.

[13] X. Wang and H. V. Poor. Blind adaptive interference suppression for CDMA communications based on eigenspace tracking. Conference on Information Sciences and Systems, pages 468473, Mar. 1997. Baltimore, MD.

[14] X. Wang and H. V. Poor. Subspace-based blind adaptive joint interference suppression and channel estimation in multipath CDMA channels. IEEE International Conference on Universal Personal Communications (ICUPC), pages 460-464, Oct. 1997. San Diego, CA.

[15] D. Zheng, J. Li, S. L. Miller, and E. G. Strom. An efficient codetiming estimator for DS-CDMA signals. IEEE Trans. Signal Processing, 45(1):82-89, Jan. 1997. 